\title{
Midgut neuroendocrine tumours with liver metastases: results of the UKINETS study
}

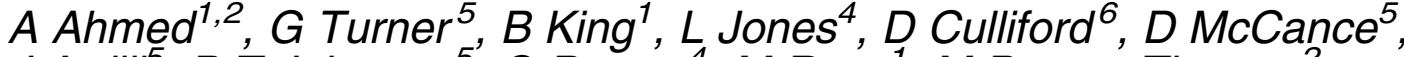

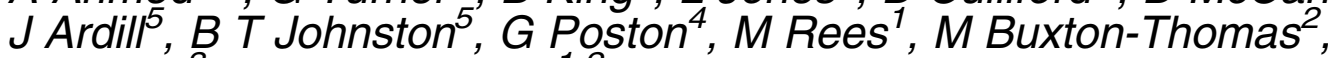 \\ M Caplin ${ }^{3}$ and J K Ramage ${ }^{1,2}$
}

\author{
${ }^{1}$ Basingstoke and North Hampshire Foundation Trust, Basingstoke, UK \\ ${ }^{2}$ Institute of Liver Studies and Department of Nuclear Medicine, Kings College Hospital, London, UK \\ ${ }^{3}$ Neuroendocrine Tumour Unit, Royal Free Hospital, London, UK \\ ${ }^{4}$ Department of Surgery, University Hospital Aintree, Liverpool, UK \\ ${ }^{5}$ Neuroendocrine Tumour Group, Royal Victoria Hospital, Belfast, UK \\ ${ }^{6}$ Research and Development Support Unit, University of Southampton, Southampton, UK
}

(Correspondence should be addressed to J K Ramage, Department of Gastroenterology, Basingstoke and North Hampshire Foundation Trust, Aldermaston Road, Basingstoke, Hampshire RG24 9NA, UK; Email: john.ramage@bnhft.nhs.uk)

\begin{abstract}
We intended to identify the prognostic factors and the results of interventions on patients with liver metastatic midgut carcinoids. Five institutions that are part of United Kingdom and Ireland neuroendocrine tumour (NET) group took part in this study. Patients were included if they had histology proven NET of midgut origin and liver metastases at the time of the study. Clinical and biochemical data were collected retrospectively from hospital charts, pathology reports, radiology reports and biochemistry records for each patient. Three hundred and sixty patients were included in the study. The median survival from date of diagnosis was 7.69 years (confidence interval $(\mathrm{Cl}) 6.40-8.99)$ and 5.95 years $(\mathrm{Cl} 5.02-6.88)$ from date of diagnosis of liver metastases. On univariate analysis, increasing age at diagnosis, increasing urinary hydroxyindole acetic acid levels, increasing plasma chromogranin A levels, high Ki67, high tumour volume and treatment with chemotherapy were identified as factors associated with a significantly poorer outcome. Resection of liver metastases, resection of small bowel primary, treatment with somatostatin analogue therapy and treatment with peptide receptor therapy were associated with improved prognosis. Multivariate analysis revealed that age at diagnosis $(P=0.014)$, Ki67 level $(P=0.039)$ and resection of primary $(P=0.015)$ were independent predictors of survival. This is the largest study to our knowledge looking specifically at the prognosis and clinical course of patients with liver metastatic midgut NETs. For the first time, we have shown that Ki67 and resection of primary are independent predictors of survival for this group of patients.

Endocrine-Related Cancer (2009) 16 885-894
\end{abstract}

\section{Background}

Midgut neuroendocrine tumours (MNETs) arise from endocrine cells of the embryological midgut. MNETs encompass tumours arising in the jejunum, ileum and proximal colon. They are associated with the development of carcinoid syndrome characterised by flushing, diarrhoea, wheezing and carcinoid heart disease. These tumours are usually at an advanced stage on presentation and are often detected at surgery for bowel obstruction, perforation or bleeding (Moertel et al. 1961, Modlin et al. 2005).

MNETs constitute the largest group of gastrointestinal neuroendocrine tumours (NETs; Modlin et al. 2003). Clarifying prognosis and guiding patients about the most appropriate course of action are difficult. This is partly due to the variable natural course of the disease, but also due to the lack of 
robust evidence of effect and outcome with the many available treatment options.

Previous studies looking at prognostic factors have shown conflicting results. The largest published study of patients with MNETs included 256 patients analysed retrospectively. One hundred and ninetyfour of these patients had liver metastases. Multivariate analysis was performed on 71 patients who had complete data. Advanced age and plasma chromogranin A $>5000 \mu \mathrm{g} / \mathrm{l}$ were significant predictors of overall survival (Janson et al. 1997).

A study of patients with metastatic MNET's was published earlier this year (Strosberg et al. 2009) with 146 patients. They found hepatic cytoreductive surgery and age were significant prognostic factors. They did not look at Ki67 as a prognostic factor. There were $21 \%$ of patients who did not have site of primary identified although a MNET was suspected. The primary was resected in 100 patients and hence only 16 patients who had a primary identified did not have resection. Resection of primary was not associated with survival benefit in this study. Multivariate analysis results have not been presented.

In another study of 399 patients, $37 \%$ of the patients were MNET's. This study found improved prognosis with primary tumours $<2.5 \mathrm{~cm}$, absence of liver metastases, absence of carcinoid symptoms and a low Ki67 (Pape et al. 2008). Two studies have identified that the prognosis of patients with MNETs is dependent on gender and presence of liver metastases (Burke et al. 1997, Tomassetti et al. 2006). One further study has identified that plasma neurokinin A was the only independent predictor of outcome (Turner et al. 2006). All studies have included patients without liver metastases. In addition, the above studies did not look at the effect of therapeutic interventions on their cohort of patients. The largest registry study (Surveillance, epidemiology and end results database) does not categorise patients with liver metastatic disease specifically.

There have been several studies, which have investigated the role of therapeutic interventions for liver metastases. These studies have all grouped foregut, midgut and hindgut tumours together during analysis (Makridis et al. 1990, Chamberlain et al. 2000, Gupta et al. 2003, Sarmiento et al. 2003, Sun et al. 2005). It is difficult to know how much of the data from these studies are relevant to clinicians treating patients with MNETs and liver metastases.

We aimed to document the clinical course and outcomes for a large group of patients with MNETs and liver metastases in order to relate these to clinicopathological variables and to the various interventions undergone by these patients.

\section{Methods}

Five institutions of the UKI NET group took part in the study. Three hundred and sixty patients were included. All patients had MNETs and liver metastases at the time of inclusion into the study. The date of diagnosis of MNETS in our cohort of patients ranged from 1973 to 2007 with 328 patients had been diagnosed since 1990. All patients had histological confirmation based on resection of primary or on biopsy of liver metastases. Histological diagnosis was based on the microscopy and immunohistochemical staining with the neuroendocrine markers.

Site of primary and assessment of metastatic disease were based on operative, cross-sectional or nuclear imaging. Forty-one patients with liver metastases and carcinoid syndrome and unknown primary were included in the study. In these cases, the clinicians felt that it was a likely midgut primary based on clinical presentation, imaging characteristics and tumour markers. These patients were not included in the analysis of survival related to resection of primary or on multivariate analysis.

\section{Data collection}

Patients were identified from existing databases at each unit. Clinical and biochemical data were collected retrospectively from hospital charts, pathology reports and biochemistry laboratory records for each patient. A specific database was designed using Filemaker Pro with drop-down menus to ensure consistency of data. This database was extensive and included prognostic factors, co-morbidities at diagnosis, extent of tumour spread and symptomatology. Additionally, all therapeutic interventions and progression of the tumour(s) were recorded annually for each year of survival of the patient from date of diagnosis of liver metastases.

To further ensure consistency, the data were collected by a single investigator A A at all centres with the exception of Royal Victoria Hospital. Here, data were collected in a separate excel-based database by $\mathrm{G} \mathrm{T}$ with the same prognostic fields and transferred directly into the Filemaker database. All attempts were made to ensure the quality of data with all medical and electronic records accessed. Primary care clinicians were also contacted when necessary.

\section{Data analysis}

Survival was measured from the date of diagnosis. In addition, survival analysis from the date of diagnosis of liver metastases and from the date of intervention for all therapies was performed. This was to document 
any survival advantage conferred by the intervention and to allow comparison with the previous similar studies. Analysis from the date of diagnosis of liver metastases was to eliminate bias of patients presenting with small bowel obstruction and the resultant early diagnosis as opposed to delayed presentation with liver metastases and carcinoid syndrome. The presence of carcinoid syndrome was defined as any of the following: flushing alone or flushing with diarrhoea or palpitations. Data on the presence of functional syndrome was available in 294 patients. Death due to all causes or date of last follow-up was used as the clinical end point. The first available biochemical data were used in the analysis for urinary 5 hydroxyindole acetic acid (HIAA) and chromogranin A.

\section{Statistical analysis}

All analysis was performed using SPSS version 15 . Initial analyses were performed to identify factors associated with the increased mortality. Comparison of values between the groups was made using MannWhitney $U$ test and $\chi^{2}$-test. In addition to age, sex, Ki67, urinary 5 HIAA, chromogranin A and all interventions, analysis was also performed on prognosis related to uptake on radio nucleotide imaging and tumour volume at presentation. For the latter analysis, patients were divided into five groups based on the presentation tumour volume: no metastases, largest metastases greater or lesser than $4 \mathrm{~cm}$ and the presence of unilobar or bilobar metastases.

Survival analysis for interventions was performed using Kaplan-Meier survival plots and comparisons between groups made with the log-rank test. Covariates identified as having a significant influence on survival by univariate analysis were included in a multivariate analysis using a Cox's proportional hazards model. Treatment with chemotherapy was not included in multivariate analysis as only four patients treated with chemotherapy had complete data for analysis.

\section{Results}

Out of the 360 patients, $189(52.5 \%)$ were male. Median age at diagnosis was 61.54 years (16-86 years). Three hundred and nine patients had an ileal primary, six had jejunal primaries and four primaries were in the caecal region. Two hundred and eighty-five patients had liver metastases at diagnosis. The mean duration of follow-up from diagnosis was 5.29 years.

One hundred and fifty-nine patients died during follow-up. Median survival from date of diagnosis was
$7.69 \pm 0.6$ years S.E.M. The 5-year survival rate from the date of diagnosis was $65.9 \%$ (Fig. 1). Median survival from the diagnosis of liver metastases was $5.95 \pm 0.47$ years (s.E.M). The 5-year survival was $56 \%$ from diagnosis of liver metastases. For causes of death see Table 1.

On univariate analysis, the following clinicopathological features were adversely related to prognosis: increasing urinary 5 HIAA $(P=0.001)$, increasing serum chromogranin A $(P=0.007)$, high Ki67 index $(P=0.005)$ and increasing age $(P=0.017)$. Patients with bilobar metastases and the largest metastases $>4 \mathrm{~cm}$ had a significantly worse outcome than all other groups of patients as divided based on tumour volume $(P<0.001)$.

There was no difference in survival for the following factors: sex $(P=0.13)$, presence of liver metastases at presentation (no metastases, unilobar, bilobar; $P=0.073)$, uptake on Octreoscan $(P=0.91)$ or on meta-iodobenzylguanidine (MIBG) scan $(P=0.23)$. Two hundred and forty-four patients had a functional syndrome at presentation. There was no difference in survival in patients with the functional syndrome than those without (median survival 6.92 vs 8.06 years; $P=0.52$ ). Calculations of survival for liver metastases at presentation and the presence of functional syndrome were undertaken from the date of diagnosis of liver metastases as opposed to the date of diagnosis. We felt that this was a better measure of the biology of the tumour rather than to compare a more advanced disease with a less advanced disease.

On survival analysis, the following treatments were associated with the improved survival: resection of

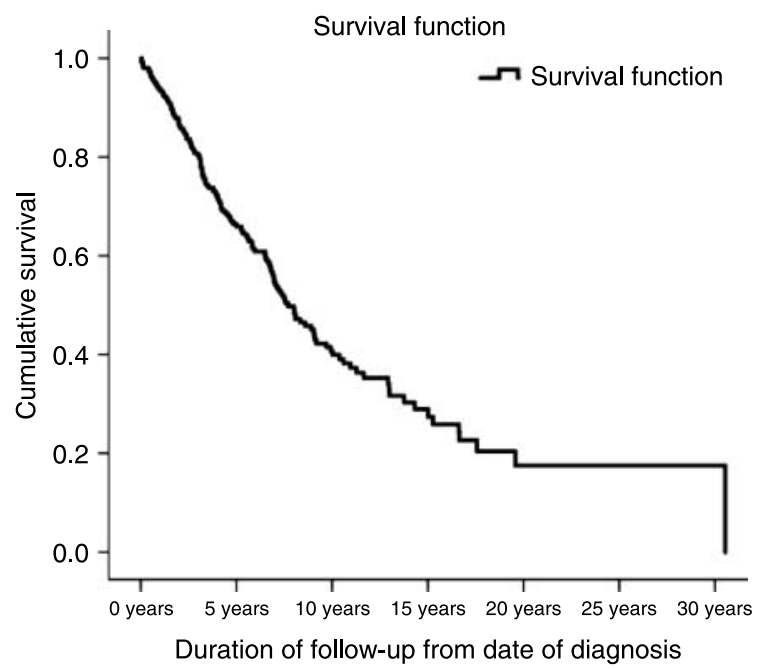

Figure 1 Kaplan-Meier survival curve of overall survival of the cohort. 
Table 1 Cause of death

\begin{tabular}{lcc}
\hline Cause of death & Number & $\begin{array}{c}\text { Percentage of } \\
\text { all deaths (\%) }\end{array}$ \\
\hline Tumour progression & 75 & 48 \\
Small bowel obstruction & 24 & 15 \\
Carcinoid heart disease & 11 & 7 \\
Intervention related & 15 & 9 \\
$\quad$ (30 day mortality) & & \\
Chemoembolisation/ & 7 & \\
$\quad$ embolisation & & \\
Peptide receptor therapy & 2 & \\
Tricuspid valve replacement & 2 & \\
$\quad$ Small bowel surgery & 3 & 11 \\
$\quad$ Chemotherapy & 1 & \\
Tumour-unrelated causes & 18 & \\
Unknown causes & 16 & \\
\hline
\end{tabular}

liver metastases, resection of small bowel primary, treatment with somatostatin analogues (SMAs) and treatment with peptide receptor therapy. Chemotherapy was associated with a significantly worse survival. There was no difference in survival in patients treated with hepatic chemoembolisation/embolisation when compared with those who did not.

\section{Prognostic factors}

Multivariate analysis was performed using Cox regression analysis, adjusting for all factors that showed a significant difference on univariate analysis. Age at diagnosis, Ki67 index and resection of primary were identified as the only independent predictors of survival (see Table 2).

\section{Resection of primary}

Two hundred and nine patients had their primary removed. Twelve patients had bowel bypass surgery. Seventeen patients had an attempted resection but were found to be irresectable. Three patients died within 30 days of surgery $(1.43 \%)$. Ten $(4.78 \%)$ patients who had resection of their primary died due to small bowel

Table 2 Results of Cox regression analysis of significant factors on univariate analysis

\begin{tabular}{lcc}
\hline Variable & $\begin{array}{c}\text { Relative } \\
\text { risk }(95 \% \mathrm{Cl})\end{array}$ & $\boldsymbol{P}$ \\
\hline Age at diagnosis & $1.07(1.015-1.146)$ & 0.014 \\
Ki67 & $1.04(1.002-1.086)$ & 0.039 \\
Resection of primary & $0.26(0.092-0.777)$ & 0.015 \\
Urinary HIAA & $1.00(1.000-1.001)$ & 0.236 \\
Serum chromogranin A & $1.00(0.998-1.002)$ & 0.923 \\
Hepatic resection & Unable to calculate & 0.988 \\
Peptide receptor therapy & $0.63(0.227-1.793)$ & 0.340 \\
Somatostatin analogue therapy & $0.41(0.045-3.737)$ & 0.478 \\
\hline
\end{tabular}

obstruction-related cachexia. Fourteen patients $(12.72 \%)$ within the three groups of 'no resection of primary', 'bypass surgery' or 'failed resection' died due to small bowel obstruction-related cachexia. There was a significant survival advantage for patients with resected primary both on univariate and multivariate analyses. The median survival for the groups was as follows: resected primary 9.92 years (confidence interval (CI) 7.45-12.40); no resection 4.68 years (CI 2.65-6.71); bowel bypass 5.61 years (CI 1.58-9.64); and failed resection 6.74 years (CI 3.32-10.16). See Table 3 for subgroup analysis between the two groups and Fig. 2 for Kaplan-Meier survival curve.

\section{Ki67 index}

Ki67 index was first described to have prognostic significance in pancreatic NETs in 1992 (Pelosi et al. 1992). Its availability in routine clinical practice in the UK was in the early part of this century. In view of a significant proportion of patients diagnosed prior to this period, data on Ki67 proliferative index were available in only 112 patients. Histology was either from the primary or the liver metastases. This specimen was usually taken at the time of diagnosis.

Patients were divided into three groups, those with Ki67 $\leq 2$ and those between 3 and 20 and those above 20 (Rindi et al. 2007). There was a significant survival advantage for patients who had low Ki67 on univariate analysis (see Fig. 3) and multivariate analysis $(P=0.039)$. The median survivals for the three groups were 16.62 years (CI $0.20-33.03$ ), 9.09 years (CI $3.67-14.51$ ) and 1.09 years (CI $0.66-1.52$ ).

\section{Medical therapy}

Two hundred and eighty-eight patients were treated with SMA therapy. Only 17 patients had had interferon therapy for any duration of time. Twelve patients had both SMA and interferon therapy who were excluded from the analysis of survival based on SMA therapy. The mean duration of treatment with SMA was 43.15 months. Treatment was generally well tolerated. There were no deaths related to the drug. Patients with the functional syndrome at presentation were more likely to have treatment with SMA therapy. In addition, patients who received SMA therapy had a significantly higher median 5 HIAA (Table 3). Survival analysis revealed that patients who had SMA therapy had a significant survival advantage when compared with those who did not $(P=0.004)$. The median survival for patients treated with SMA was 8.06 years (CI 6.30-9.83) versus 5.6 years (CI 3.34-7.87) for those who did not. Given the retrospective nature of the study, it was difficult to 
Table 3 Intervention-related subgroup analysis

\begin{tabular}{lcccccc}
\hline & $\begin{array}{c}\text { Mean age } \\
\text { (years) }\end{array}$ & $\begin{array}{c}\text { Median Ki67 } \\
\text { index }\end{array}$ & $\begin{array}{c}\text { Median } \\
\text { 5 HIAA }\end{array}$ & $\begin{array}{c}\text { Median } \\
\text { CGA }\end{array}$ & $\begin{array}{c}\text { Median } \\
\text { survival (years) }\end{array}$ & $\begin{array}{c}\text { 5-year } \\
\text { survival (\%) }\end{array}$ \\
\hline Resected primary & 59.94 & 1.00 & 156.0 & 203.5 & 9.92 & 74 \\
No resection & 60.67 & 5.00 & 392.5 & 531.0 & 4.73 & 46 \\
Hepatic surgery & 58.35 & 5.91 & 226.0 & 163.5 & $11.26^{\mathrm{a}}$ & $78^{\mathrm{a}}$ \\
No hepatic surgery & 61.97 & 2.00 & 229.0 & 285.0 & $5.50^{\mathrm{a}}$ & $52^{\mathrm{a}}$ \\
SMA therapy & 59.67 & 2.00 & 270.0 & 282.0 & 8.06 & 50.6 \\
No SMA therapy & 62.27 & 2.00 & 115.0 & 310.0 & 5.60 & 68.5 \\
HAE/HACE & 56.01 & 5.00 & 361.5 & 476.0 & $5.50^{\mathrm{a}}$ & $52.7^{\mathrm{a}}$ \\
No HAE/HACE & 60.99 & 2.00 & 217.0 & 280.0 & $6.52^{\mathrm{a}}$ & $57.3^{\mathrm{a}}$ \\
Peptide receptor therapy & 57.96 & 5.00 & 330.0 & 282.0 & $6.98^{\mathrm{a}}$ & $66.4^{\mathrm{a}}$ \\
No peptide receptor therapy & 61.17 & 2.00 & 194.5 & 285.0 & $5.51^{\mathrm{a}}$ & $50.5^{\mathrm{a}}$ \\
Chemotherapy & 56.67 & 10.0 & 226.0 & 1000.0 & $2.30^{\mathrm{a}}$ & $40.7^{\mathrm{a}}$ \\
No chemotherapy & 60.44 & 2.00 & 229.0 & 281.0 & $6.33^{\mathrm{a}}$ & $57.2^{\mathrm{a}}$ \\
\hline
\end{tabular}

${ }^{a}$ Analysis from the date of diagnosis of liver metastases.

be certain as to whether the initiation of SMA was for its antiproliferative or symptom control effects. Data with regards to this were hence not collected.

\section{Hepatic resection}

Fifty patients underwent hepatic resection for their liver metastases. Out of these, three underwent resection twice due to recurrence and one patient underwent resection three times. A significantly higher proportion had their primary resected $(90.7 \%)$ in the hepatic surgery group as opposed to $61.6 \%$ in the no hepatic surgery group. This relationship between hepatic resection and resection of primary resulted in the inability to calculate relative risk of hepatic resection in multivariate analysis.

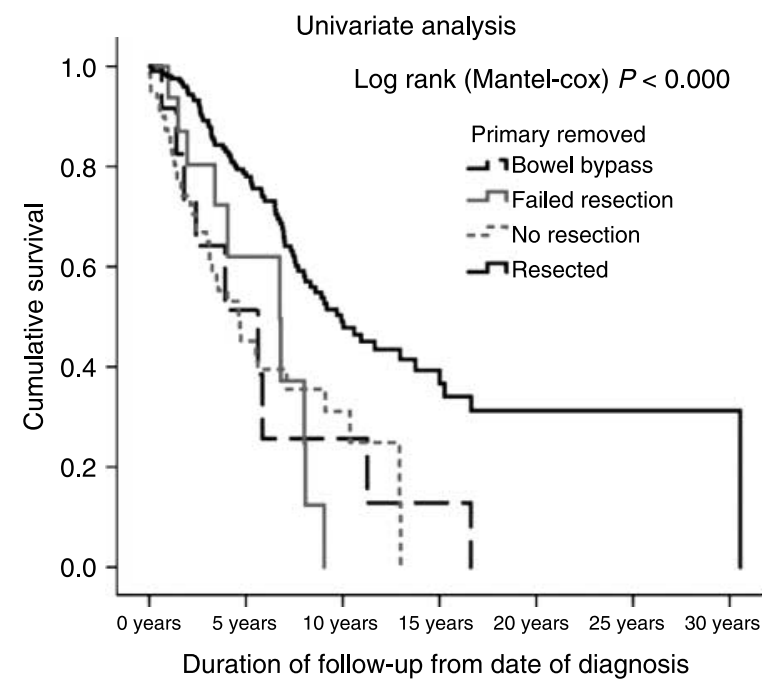

Figure 2 Survival of patients with bowel bypass versus failed resection versus no resection versus resection. $n=12 / 17 / 80 / 210$.
The 5-year survival from resection of liver metastases was $74.3 \%$. There were no surgery-related deaths. On univariate analysis, patients who underwent hepatic resection had a significant survival benefit when compared with those who did not. The median survival was 11.26 years (CI 5.19-17.33) for those who had resection versus 5.50 years (CI 4.33-6.17) for those who did not. See Table 3 for subgroup analysis and Fig. 4 for Kaplan-Meier survival curve.

\section{Hepatic embolisation/chemoembolisation}

Forty-two patients underwent hepatic artery embolisation (HAE) and 14 patients underwent hepatic artery chemoembolisation (HACE). Out of these 56 patients, 36 underwent one episode, 12 underwent two episodes, 7 underwent three episodes and 1 patient underwent four episodes. Seven patients died within 30 days of a procedure. Six deaths were following chemoembolisation and one following embolisation. Five of these patients died on their first episode and one each on the second and third episode. The median survival from the date of first procedure was 3.2 \pm 0.51 years (s.E.M). The 30 -day mortality was $12.5 \%$. The 5-year survival from the date of first procedure was $29 \%$. In addition to having a higher mean 5 HIAA and CGA levels, patients who underwent HAE/HACE had a higher proportion of patients with greater tumour volume as classified previously (group 5: 53 vs 31\%). The median survival for patients who had chemo/embolisation was 5.50 years (CI 3.77-7.23) versus 6.53 years (CI 5.39-7.64) for those who did not. There was no difference in survival for patients who underwent hepatic chemo/ embolisation versus those who did not $(P=0.321)$. See Table 3 for subgroup analysis. 


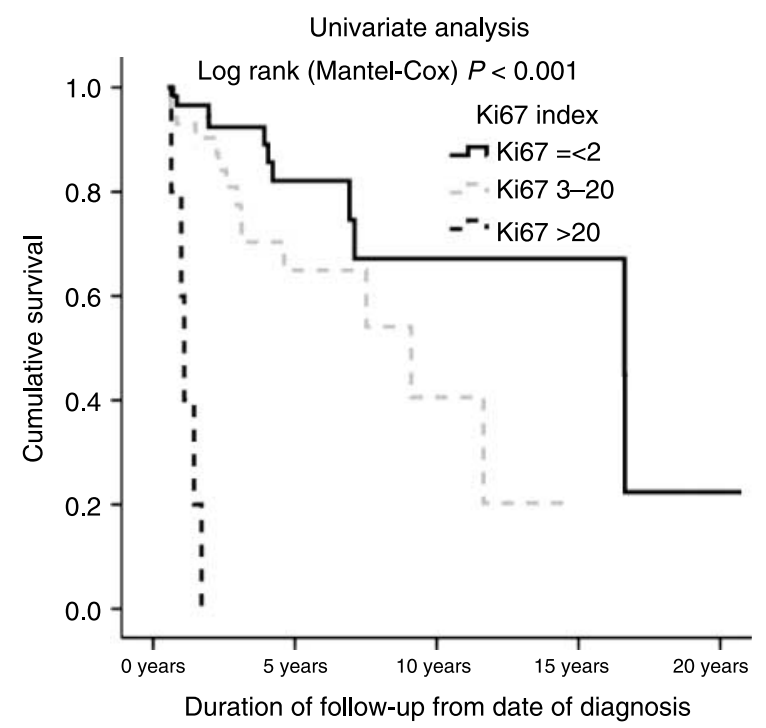

Figure 3 Survival differences between patients with Ki67 $\leq 2$, $3-20$ and $>20$. $n=60 / 45 / 6$.

\section{Peptide receptor therapy}

Octreoscans were performed on 267 patients and MIBG scans were performed on 227 patients. There were 217 patients $(81.2 \%)$ in whom octreoscan uptake in the metastases was greater than the background liver. This suggests that a large proportion of these patients would be eligible for peptide receptor therapy. Seventy-three patients underwent MIBG therapy, 21 patients underwent Yttrium 90 dotatoc therapy, 8 underwent Yttrium SMT 480 therapy and 8 patients underwent Indium 111 Octreotide therapy. Twentyfour patients had a single therapeutic episode, 35

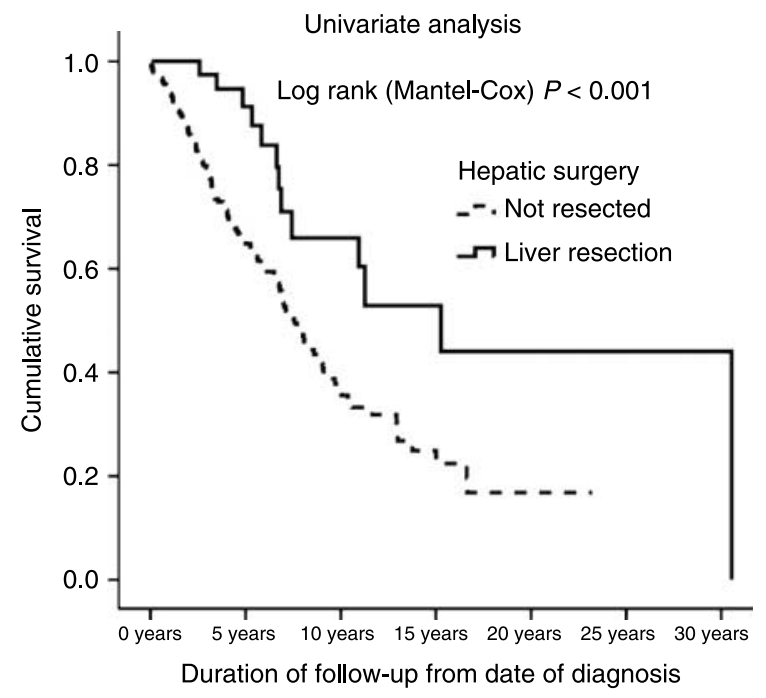

Figure 4 Survival of patients with no hepatic resection versus hepatic resection. $n=310 / 50$. patients had two therapeutic episodes, 31 patients had three therapeutic episodes and 10 patients had four therapeutic episodes. A total of 290 episodes of peptide receptor therapy were given to 120 patients. Two patients died within 30 days of their peptide receptor therapy $(1.9 \%)$. The median survival from the first episode of peptide receptor therapy was $4.5 \pm 0.67$ years (S.E.M). The 5-year survival post therapy was $40 \%$. The median survival for patients who had therapy was 9.04 years (CI 6.47-11.61) versus 7.21 years (CI 5.91-8.50) for those who did not. There was significant survival difference on univariate analysis $(P=0.007)$. See Table 3 for subgroup analysis.

\section{Chemotherapy}

Twenty-four patients had chemotherapy for their liver metastatic MNET. Majority of the patients had streptozocin and 5-fluorouracil-based therapy. A few patients had cisplatin or lomustine. One patient died within 30 days of therapy. Five-year survival post therapy was $22.7 \%$. The median survival for patients who had chemotherapy was 2.79 years (CI 0-6.58) versus 6.33 years (CI 5.50-7.17) for those who did not. As is noted in Table 3, patients who had chemotherapy had a higher mean CGA. There was no difference in tumour volumes between the two groups (group 5: 35 vs $33 \%$.) Univariate analysis revealed that patients who had chemotherapy had significantly poorer outcome compared with those who did not $(P=0.005)$.

\section{Discussion}

This is the largest study to our knowledge specifically looking at patients with liver metastatic MNETs. Despite its retrospective nature, confounding factors of previous studies have been eliminated by selecting a specific cohort of patients and modelling for all known prognostic variables. Many more clinical, radiological and biochemical factors relating to prognosis were analysed than any other paper in this group of patients. In addition, their significance was assessed in relation to the therapeutic interventions patients underwent.

This study suggests, for the first time, that resection of primary and Ki67 are independent predictors of survival in patients with MNETs with liver metastases. These factors remained significant if multivariate analysis was performed from the date of diagnosis of liver metastases as opposed to the date of diagnosis of the tumour.

A previous retrospective study has shown improved survival of patients undergoing debulking of primary in midgut tumours (Soreide et al. 1992). Unlike this 
study, multivariate analysis was not done and hence the bias for known prognostic factors and interventions was not undertaken. Stosberg et al. have recently found no survival benefit in resection of primary in metastatic MNET's; however, the number of patients who did not have resection was too small to make any firm conclusion.

In this study, it is possible that fitter patients were selected for resection of primary. In addition, the survival rates were similar in patients who had bowel bypass, failed resection and no resection. This suggests that although the patients were fit enough to undergo surgery in the former groups, the failure to resect the primary adversely affected their prognosis. Despite the differences in the subgroup analysis shown in Table 3, resection of primary was the only intervention which remained significant on multivariate analysis.

In view of the significantly higher proportion of patients dying as a result of small bowel obstruction in unresected patients, the low procedure-related mortality $(1.43 \%)$ and the demonstrated survival advantage we feel there may be at least some evidence that patients with MNETS and liver metastases should have their primary resected if technically feasible. A randomised controlled trial of resection of primary should now be considered. These data also confirm suspicions from other studies that increasing age (Janson et al. 1997) and higher Ki-67 (Rosenau et al. 2002, Amarapurkar et al. 2003, Tomassetti et al. 2006) result in poorer survival.

Other factors that are only significant on univariate analysis are more difficult to interpret and will be considered below. Patients with increasing urinary 5 HIAA, increasing serum chromogranin A and bilobar liver metastases (largest $>4 \mathrm{~cm}$ at presentation) had a poorer prognosis. This endorses previous studies (Janson et al. 1997). However, interventions have been shown to affect these factors (Ardill \& Erikkson 2003, Sondenaa et al. 2004, Akerstrom et al. 2005). This study shows that when interpreted in the context of interventions, none of these factors remain statistically significant.

Data on results of patients undergoing hepatic resection were similar to the largest study by Sarmiento et al. with $61 \%$ 5-year survival and $1.2 \%$ mortality. It is likely that there was a bias in these patients, where clinicians selected patients perhaps based on their age, fitness and/or Ki67. However, patients who are suitable for liver resection should continue to be offered this therapy since there is a low procedure-related mortality and it improves quality of life by reducing carcinoid symptoms (McEntee et al. 1990, Chamberlain et al. 2000).
In a randomised prospective study in this group, 80 patients (30 MNETs) were randomised to treatment with lanreotide, interferon $\alpha$ and combination therapy. All treatment strategies showed similar rates of tumour stabilisation (18-28\%; Faiss et al. 2003). This rate is lower than previous nonrandomised retrospective studies (24-57\%; Kvols et al. 1986, Oberg et al. 1991, Jacobsen \& Hanssen 1995, Di Bartolomeo et al. 1996, Eriksson et al. 1997, Ducreux et al. 2000, Welin et al. 2004). These results have been replicated in a recent yet to be published randomised prospective study of patients with metastatic MNET's. Here, time to progression was significantly higher (14.3 vs 6 month's) in patients randomised to octreotide LAR versus placebo (Arnold et al. 2009). This study has demonstrated a survival advantage for patients treated with SMA on univariate analysis. If anything, patients with more advanced disease were more likely to be treated with SMA. However, since multivariate analysis was not significant, the case for SMA prolonging life is unproven.

The 5-year survival of patients who had HACE/ HAE is similar to the $28.6 \%$ reported by Gupta et al (2003) in 123 patients. However, other studies have reported better survival rates such as $60 \%$ by Eriksson et al. (1998). In this study of 29 patients, gel foam embolisation was performed rather than HACE. The early mortality rates from previous studies have varied from 0 to $8.7 \%$ (Chamberlain et al. 2000, Yao et al. 2001, Kress et al. 2003, Loewe et al. 2003). In our study, six out of the seven deaths were patients who had HACE. This suggests that HAE may be safer. Embolisation techniques have also improved with the selective embolisation now routinely available. In addition, radioembolisation with 90Y-microspheres, a technology similar to HAE/HACE, has shown significant promise (Kennedy et al. 2008). This strategy therefore remains a useful adjunct in palliating patients with the carcinoid symptoms that are difficult to control, but patients should be informed that there is no proven survival advantage.

Peptide receptor therapy results are similar to other previously reported studies (Waldherr et al. 2002, Bomanji et al. 2003, Safford et al. 2004, Sywak et al. 2004). A relatively new agent Lu177-DOTATATE has now been shown to have better survival data with the median survival of 46 months from the start of treatment and 128 months form the date of diagnosis (Kwekkeboom et al. 2008). To date, patients are more likely to have peptide receptor therapy if they are unfit for other interventions and have more advanced disease. This would tend to make the difference on 
univariate analysis less prone to bias, but multivariate analysis did not reach statistical significance.

Patients who had chemotherapy did worse than those who did not in univariate analysis. The median survival rates were similar to the only other randomised study in this group of patients. Here, 176 patients (43 MNETs) were randomised to fluorouracil and doxazosin versus fluorouracil and streptozocin (Sun et al. 2005). There is likely to be at least in part a negative bias in that these patients are often end stage with an advanced disease. Several newer agents such as tyrosine kinase inhibitors, angiogenic inhibitors and mammalian target of rapamycin inhibitors are currently being investigated, which have shown promise in early studies. These agents may offer an effective chemotherapeutic strategy in the future. However, on the basis of the above data, there is no proven role for chemotherapy in prolonging life in patients with midgut NETs with liver metastases.

In the absence of randomised trials for measuring the survival benefit of established interventions, these data for the first time give the best current guide to the value of treatment options in this very specific group of patients. This may help clinicians and patients in making difficult therapeutic decisions. It also provides detailed clinical data necessary for cost effective analysis and direction for future clinical research.

\section{Declaration of interest}

We declare that there is no conflict of interest that could be perceived as prejudicing the impartiality of the research reported.

\section{Funding}

This work was supported in part by unrestricted grant from Novartis UK, Ipsen Ltd and the MAPI Institute.

\section{Author contribution statement}

A Ahmed collected data from Basingstoke and North Hampshire NHS Trust, Kings College Hospital, Royal Free Hospital and University Hospital Aintree. G Turner collected data from Neuroendocrine Tumour Group Royal Victoria Hospital, Belfast. D Culliford provided statistical support for all analysis. All other authors are clinicians responsible for patient's management and contributed to editing the paper prior to submission.

\section{References}

Akerstrom G, Hellman P, Hessman O \& Osmak L 2005 Management of midgut carcinoids. Journal of Surgical Oncology 89 161-169.
Amarapurkar AD, Davies A, Ramage JK, Stangou AJ, Wight DG \& Portmann BC 2003 Proliferation of antigen MIB-1 in metastatic carcinoid tumours removed at liver transplantation: relevance to prognosis 141 . European Journal of Gastroenterology \& Hepatology 15 139-143. Ardill JE \& Erikkson B 2003 The importance of the measurement of circulating markers in patients with neuroendocrine tumours of the pancreas and gut. Endocrine-Related Cancer 10 459-462.

Arnold R, Müller H, Schade-Brittinger C, Rinke A, Klose K, Barth P, Wied M, Mayer C \& Aminossadati B, PROMID Study Group 2009 Placebo-controlled, double-blind, prospective, randomized study of the effect of octreotide LAR in the control of tumor growth in patients with metastatic neuroendocrine midgut tumors: a report from the PROMID study group. Journal of Clinical Oncology 27 (Supp) 4508.

Di Bartolomeo M, Bajetta E, Buzzoni R, Mariani L, Carnaghi C, Somma L, Zilembo N \& Di Leo A 1996 Clinical efficacy of octreotide in the treatment of metastatic neuroendocrine tumors. A study by the Italian Trials in Medical Oncology Group. Cancer 77 402-408.

Bomanji JB, Wong W, Gaze MN, Cassoni A, Waddington W, Solano J \& Ell P 2003 Treatment of neuroendocrine tumours in adults with ${ }^{131}$ I-MIBG therapy. Clinical Oncology 15 193-198.

Burke AP, Thomas RM, Elsayed AM \& Sobin LH 1997 Carcinoids of the jejunum and ileum: an immunohistochemical and clinicopathologic study of 167 cases. Cancer 79 1086-1093.

Chamberlain RS, Canes D, Brown KT, Saltz L, Jarnagin W, Fong Y \& Blumgart LH 2000 Hepatic neuroendocrine metastases: does intervention alter outcomes? Journal of the American College of Surgeons 190 432-445.

Ducreux M, Ruszniewski P, Chayvialle JA, Blumberg J, Cloarec D, Michel H, Raymond JM, Dupas JL, Gouerou $\mathrm{H}$, Jian R et al. 2000 The antitumoral effect of the longacting somatostatin analog lanreotide in neuroendocrine tumors. American Journal of Gastroenterology 95 3276-3281.

Eriksson B, Renstrup J, Imam H \& Oberg K 1997 High-dose treatment with lanreotide of patients with advanced neuroendocrine gastrointestinal tumors: clinical and biological effects. Annals of Oncology 8 1041-1044.

Eriksson BK, Larsson EG, Skogseid BM, Lofberg AM, Lorelius LE \& Oberg KE 1998 Liver embolizations of patients with malignant neuroendocrine gastrointestinal tumors. Cancer 83 2293-2301.

Faiss S, Pape UF, Bohmig M, Dorffel Y, Mansmann U, Golder W, Riecken EO \& Wiedenmann B 2003 Prospective, randomized, multicenter trial on the antiproliferative effect of lanreotide, interferon alfa, and their combination for therapy of metastatic neuroendocrine gastroenteropancreatic tumors - the International Lanreotide and Interferon Alfa Study Group. Journal of Clinical Oncology 21 2689-2696. 
Gupta S, Yao JC, Ahrar K, Wallace MJ, Morello FA, Madoff DC, Murthy R, Hicks ME \& Ajani JA 2003 Hepatic artery embolization and chemoembolization for treatment of patients with metastatic carcinoid tumors: the M.D. Anderson experience. Cancer Journal 9 261-267.

Jacobsen MB \& Hanssen LE 1995 Clinical effects of octreotide compared to placebo in patients with gastrointestinal neuroendocrine tumours: report on a doubleblind, randomized trial. Journal of Internal Medicine $\mathbf{2 3 7}$ 269-275.

Janson ET, Holmberg L, Stridsberg M, Eriksson B, Theodorsson E, Wilander E \& Oberg K 1997 Carcinoid tumors: analysis of prognostic factors and survival in 301 patients from a referral center. Annals of Oncology 8 685-690.

Kennedy AS, Dezarn WA, McNeillie P, Coldwell D, Nutting C, Carter D, Murthy R, Rose S, Warner RR, Liu D et al. 2008 Radioembolization for unresectable neuroendocrine hepatic metastases using resin 90Y-microspheres: early results in 148 patients. American Journal of Clinical Oncology 31 271-279.

Kress O, Wagner HJ, Wied M, Klose KJ, Arnold R \& Alfke H 2003 Transarterial chemoembolization of advanced liver metastases of neuroendocrine tumors - a retrospective single-center analysis. Digestion 68 94-101.

Kvols LK, Moertel CG, O’Connell MJ, Schutt AJ, Rubin J \& Hahn RG 1986 Treatment of the malignant carcinoid syndrome Evaluation of a long-acting somatostatin analogue157. New England Journal of Medicine 315 663-666.

Kwekkeboom DJ, de Herder WW, Kam BL, van Eijck CH, van Essen M, Kooij PP, Feelders RA, van Aken MO \& Krenning EP 2008 Treatment with the radiolabeled somatostatin analog [177 Lu-DOTA 0,Tyr3]octreotate: toxicity, efficacy, and survival. Journal of Clinical Oncology 26 2124-2130.

Loewe C, Schindl M, Cejna M, Niederle B, Lammer J \& Thurnher S 2003 Permanent transarterial embolization of neuroendocrine metastases of the liver using cyanoacrylate and lipiodol: assessment of mid- and long-term results. American Journal of Roentgenology $\mathbf{1 8 0}$ 1379-1384.

Makridis C, Oberg K, Juhlin C, Rastad J, Johansson H, Lorelius LE \& Akerstrom G 1990 Surgical treatment of mid-gut carcinoid tumors. World Journal of Surgery 14 377-383.

McEntee GP, Nagorney DM, Kvols LK, Moertel CG \& Grant CS 1990 Cytoreductive hepatic surgery for neuroendocrine tumors 1. Surgery 108 1091-1096.

Modlin IM, Lye KD \& Kidd M 2003 A 5-decade analysis of 13,715 carcinoid tumors 36. Cancer 97 934-959.

Modlin I, Kidd M, Latich I, Zikusoka M \& Shapiro M 2005 Current status of gastrointestinal carcinoids 3 . Gastroenterology 128 1717-1751.

Moertel CG, Sauer WG, Dockerty MB \& Baggenstoss AH 1961 Life history of the carcinoid tumor of the small intestine 25. Cancer 14 901-912.
Oberg K, Norheim I \& Theodorsson E 1991 Treatment of malignant midgut carcinoid tumours with a long-acting somatostatin analogue octreotide. Acta Oncologica 30 503-507.

Pape UF, Berndt U, Muller-Nordhorn J, Bohmig M, Roll S, Koch M, Willich SN \& Wiedenmann B 2008 Prognostic factors of long-term outcome in gastroenteropancreatic neuroendocrine tumours. Endocrine-Related Cancer 15 1083-1097.

Pelosi G, Zamboni G, Doglioni C, Rodella S, Bresaola E, Iacono C, Serio G, Iannucci A \& Scarpa A 1992 Immunodetection of proliferating cell nuclear antigen assesses the growth fraction and predicts malignancy in endocrine tumors of the pancreas. American Journal of Surgical Pathology 16 1215-1225.

Rindi G, Kloppel G, Couvelard A, Komminoth P, Korner M, Lopes J, McNicol AM, Nilsson O, Perren A, Scarpa A et al. 2007 TNM staging of midgut and hindgut (neuro) endocrine tumors: a consensus proposal including a grading system. Virchows Archiv 451 757-762.

Rosenau J, Bahr M, von W, Mengel M, Schmidt H, Nashan Br, Lang H, Klempnauer J, Manns M \& Boeker K 2002 Ki67, E-cadherin, and p53 as prognostic indicators of long-term outcome after liver transplantation for metastatic neuroendocrine tumors. Transplantation 73 386-394.

Safford SD, Coleman RE, Gockerman JP, Moore J, Feldman J, Onaitis MW, Tyler DS \& Olson JA Jr 2004 Iodine-131 metaiodobenzylguanidine treatment for metastatic carcinoid. Results in 98 patients. Cancer 101 1987-1993.

Sarmiento JM, Heywood G, Rubin J, Ilstrup DM, Nagorney DM \& Que FG 2003 Surgical treatment of neuroendocrine metastases to the liver: a plea for resection to increase survival. Journal of the American College of Surgeons 197 29-37.

Sondenaa K, Sen J, Heinle F, Fjetland L, Gudlaugsson E \& Syversen U 2004 Chromogranin A, a marker of the therapeutic success of resection of neuroendocrine liver metastases: preliminary report. World Journal of Surgery 28 890-895.

Soreide O, Berstad T, Bakka A, Schrumpf E, Hanssen LE, Engh V, Bergan A \& Flatmark A 1992 Surgical treatment as a principle in patients with advanced abdominal carcinoid tumors. Surgery 111 48-54.

Strosberg J, Gardner N \& Kvols L 2009 Survival and prognostic factor analysis of 146 metastatic neuroendocrine tumors of the mid-gut. Neuroendocrinology 89 471-476.

Sun W, Lipsitz S, Catalano P, Mailliard JA \& Haller DG 2005 Phase II/III study of doxorubicin with fluorouracil compared with streptozocin with fluorouracil or dacarbazine in the treatment of advanced carcinoid tumors: Eastern Cooperative Oncology Group Study E1281. Journal of Clinical Oncology 23 4897-4904. 
Sywak M, Pasieka J, McEwan A, Kline G \& Rorstad O 2004

${ }^{131}$ I-meta-iodobenzylguanidine in the management of metastatic midgut carcinoid tumors. World Journal of Surgery 28 1157-1162.

Tomassetti P, Campana D, Piscitelli L, Casadei R, Nori F, Brocchi E, Santini D, Pezzilli R \& Corinaldesi R 2006 Endocrine tumors of the ileum: factors correlated with survival. Neuroendocrinology 83 380-386.

Turner GB, Johnston BT, McCance DR, McGinty A, Watson RG, Patterson CC \& Ardill JE 2006 Circulating markers of prognosis and response to treatment in patients with midgut carcinoid tumours. Gut 55 1586-1591.

Waldherr C, Pless M, Maecke H, Schumacher T, Crazzolara A, Nitzsche E, Haldemann A \& Mueller B 2002
Tumor response and clinical benefit in neuroendocrine tumors after $74 \mathrm{GBq} 90 \mathrm{Y}$-DOTATOC. Journal of Nuclear Medicine 43 610-616.

Welin SV, Janson E, Sundin A, Stridsberg M, Lavenius E, Granberg D, Skogseid B, Oberg K \& Eriksson B 2004 High-dose treatment with a long-acting somatostatin analogue in patients with advanced midgut carcinoid tumours. European Journal of Endocrinology 151 107-112.

Yao KA, Talamonti MS, Nemcek A, Angelos P, Chrisman H, Skarda J, Benson AB, Rao S, Joehl RJ, Demeure MJ et al. 2001 Indications and results of liver resection and hepatic chemoembolization for metastatic gastrointestinal neuroendocrine tumors. Surgery 130 677-685. 